\title{
ESTILO DE VIDA DE ESTUDANTES DE GRADUAÇÃO EM ENFERMAGEM DE UMA INSTITUIÇÃO DO SUL DO BRASIL
}

\author{
Everton Fernando Alves ${ }^{1}$ \\ ${ }^{1}$ Clínica de Cirurgia Plástica Dr. Fábio Paixão
}

\section{RESUMO}

Objetivou-se analisar o estilo de vida de estudantes de enfermagem por meio de um estudo exploratório, descritivo e com abordagem quantitativa. Verificou-se que os estudantes ingeriam apenas dois grupos de alimentos ("verduras e frutas" ou "verduras e grãos"); $54,2 \%$ não praticava atividade física; $57,8 \%$ deles dormiam menos de sete horas/ dia; havia grande proporção de estudantes-trabalhadores; $9,9 \%$ de fumantes e a frequência do uso de tabaco foi de $52,6 \%$ fumando de 2 a 5 cigarros/ dia, e 26,3\% fumando de 1 a 3 maços de cigarros/ semana; 27,1\% de estudantes etilistas e destes, 42,3\% desses fazem o uso de álcool de 1 a 5 vezes/ semana, considerados bebedores moderados a graves. Conclui-se que, os estudantes não apresentam um estilo de vida saudável, colocando-os em risco de agravos a saúde.

Palavras-chave: Estilo de vida, Estudantes, Enfermagem.

\section{ABSTRACT}

This study aimed to analyze the lifestyle of nursing through an exploratory study, and described with quantitative approach. It was found that students ate only two food groups ("vegetables and fruits" or "vegetables and beans"); $54,2 \%$ did not practice physical activity; $57,8 \%$ of them slept less than seven hours/day; there was great proportion of student workers; $9,9 \%$ of smokers and the frequency of tobacco use was $52,6 \%$ smoked $2-5$ cigarettes/ day and $26,3 \%$ smoked $1-3$ cigaretts masses/ week; $27,1 \%$ of students consumed alcohol and of these, $42,3 \%$ do so $1-5$ times/ week, considered moderate to severe drinkers. It was concluded that students do not have ahealthy lifestyle, putting them at risk of harm to health.

Keywords: Lifestyle, Students, Nursing.

\section{INTRODUÇÃO}

A universidade se apresenta como um espaço para o ensino-aprendizagem que promove um choque de mudanças na vida de muitos jovens recém-egressos que se afastam de seus lares e de seus antigos hábitos. Dessa forma, são adquiridos e reforçados novos hábitos refletindo num modelo para o estilo que esse estudante poderá levar por toda sua vida ou neles permanecer por tempo suficiente para afetar sua saúde (SANTOS e ALVES, 2009).

O estilo de vida pode ser definido como "o conjunto de ações habituais que refletem os valores, as atitudes e oportunidades na vida das pessoas" (NAHAS, 
2003, p. 11). Mas, optar por uma vida saudável nem sempre é fácil devido influência por fatores culturais, sociais e religiosos, socioeconômicos, ambientais e educacionais. Apesar de todos esses fatores, o estilo de vida é visto como uma dimensão da Qualidade de Vida (QV) e de característica interdisciplinar (UCHOA; ROSEMBERG; PORTO, 2002).

Assim sendo, em diversos cursos da saúde, apesar de os estudantes terem consciência de que a saúde tem relação direta com a QV muitos apresentam reduzida preocupação com seus próprios comportamentos de risco, direcionando nesta fase da vida, maior atenção às questões de acumulação de bens materiais, justificando os sacrifícios de ordem pessoal para alcançar objetivos materiais (SANTOS, 2004).

Entretanto sabe-se que, uma das principais finalidades em_manter um estilo de vida com menos comportamentos de risco é prevenir ou retardar o desenvolvimento prematuro de problemas de saúde, prolongando as porções saudáveis e independentes da vida (NAHAS, 2003; COELHO e SANTOS, 2006).

Nos dias atuais, é possível que o estilo de vida seja um dos mais importantes determinantes de saúde da população estudantil. No entanto, apesar do acesso a informações de saúde a que eles possuem, os estudos comportamentais mostram que estudantes revelam cada vez mais novos comportamentos de risco e definições sobre estilo de vida (VIEIRA et al, 2002; FRANCA e COLARES, 2008; JOIA, 2010).

Os estudantes de enfermagem, especificamente, têm tido um conceito singular de estilo de vida (modo como se vive) e estilo de vida saudável (qualidade de vida); eles têm pontuado como fatores de seu estilo de vida saudável a alimentação, por exemplo, e como não-saudáveis o sedentarismo e, ainda, identificam que há mudanças no seu estilo de vida durante o período letivo como no quesito alimentação, além de outros aspectos que podem ser prejudiciais à saúde deles (SOARES e CAMPOS, 2008).

Nesse contexto, a investigação de informações a cerca do estilo de vida de estudantes de enfermagem se torna fundamental para a ampliação do sistema de informações sobre hábitos relacionados à saúde desse grupo no intuito de traçar perfis de comportamentos de risco para que as instituições de ensino superior em enfermagem possam desenvolver planejamento estratégico a fim de que se reverta possíveis quadros conflitantes, uma vez que aspectos negativos no estilo de vida 
dos estudantes podem afetar negativamente $\mathrm{o}$ desempenho acadêmico e $\mathrm{o}$ processo de ensino-aprendizagem desses futuros profissionais.

Dessa forma, objetivou-se neste estudo analisar alguns aspectos do estilo de vida de estudantes de enfermagem envolvendo dimensões relacionadas a hábitos alimentares, atividade física, padrão de sono/repouso, e comportamentos de risco tais como o uso de tabaco e álcool.

\section{METODOLOGIA}

Trata-se de um estudo exploratório com delineamento descritivo e abordagem quantitativa, tendo como população de estudo os estudantes matriculados nos quatro anos do curso de Enfermagem da faculdade Uningá, em Maringá - PR.

Como instrumento de coleta de dados utilizou-se um questionário auto-aplicável contendo questões estruturadas relacionadas a alguns componentes que permeiam o estilo de vida de um indivíduo, representados pelas variáveis: hábitos alimentares, atividade física, padrão de sono/repouso e consumo de tabaco e álcool. Limitou-se a estes aspectos, sem levar em conta fatores de herança genética, concepções políticas e outros fatores que podem influenciar os resultados.

Os procedimentos para coletas de dados incluíram: aprovação da pesquisa pelo diretor acadêmico da instituição; contato com docentes solicitando autorização e agendamento para aplicar o instrumento em sala de aula, apresentação da proposta aos alunos presente no momento da coleta, com rápida explanação sobre os objetivos do estudo, o método de coleta de dados, importância da colaboração e questões éticas envolvidas (adesão voluntária, sigilo, anonimato e contribuição do estudo). Neste momento foi apresentada e solicitada a assinatura do termo de consentimento livre e esclarecido aos participantes.

A coleta de dados foi realizada no mês de maio de 2006, sendo efetuada pelo próprio autor do trabalho. O desenvolvimento do estudo ocorreu em conformidade com o preconizado pela Resolução 196/96 do CNS. Para a análise dos dados foi utilizadas técnicas de estatística descritiva. Os dados foram digitados em planilha eletrônica e os cálculos estatísticos foram feitos no software Excel e organizados e apresentados, posteriormente, em forma de tabelas.

Para fins de análise, foi utilizada a definição de fumante da OMS apresentada no estudo de Oguisso e Seki (2001). Segundo essa definição, é considerado: 
1. Fumante - aquele que fumava na data do estudo, podendo ser classificado em: a) Fumante diário ou habitual - aquele que fuma diariamente, mesmo que eventualmente passe um dia sem fumar; b) Fumante ocasional - aquele que fuma socialmente ou em determinadas situações.

2. Não fumante - aquele que não fumava na data do estudo, podendo serser classificado em: a) Ex-fumante diário - aquele que fumava diariamente e deixou completamente de fumar; b) Ex-fumante ocasional - aquele que fumava ocasionalmente e deixou de fazê-lo e; c) Não fumante propriamente dito - nunca fumou de forma alguma e não fuma atualmente.

Para definição de estudantes etilistas, utilizou-se a classificação do Ministério da Saúde (1994). Assim sendo, considera-se: Bebedor leve - homens que consomem menos que 21 unidades de álcool por semana e mulheres que consomem até 14 unidades por semana; Bebedor moderado - homens que consomem de 22-50 unidades/semana e mulheres 15-35 unidades/semana e; Bebedor grave - homens que consomem acima de 51 unidades e mulheres acima de 36. Cada unidade de álcool, por exemplo, equivale à 10g; $350 \mathrm{ml}$ de cerveja com 4\% de álcool equivalem a 1,5 unidades de álcool. Isto quer dizer que homens podem consumir no máximo 2 latas de cerveja por dia (ao longo do dia) e mulheres 1 lata.

\section{RESULTADOS E DISCUSSÃO}

Dos 243 estudantes matriculados no curso de graduação em Enfermagem da instituição pesquisada em 2006, 194 deles participaram do estudo, visto que 45 não estavam presentes na sala de aula nos períodos destinados à coleta de dados e 04 se recusaram a participar do estudo e 02 foram excluídos por não responderem todo o instrumento de coleta de dados resultando numa amostra de 192 estudantes.

Quanto à caracterização da amostra nota-se que, a idade média dos acadêmicos foi 24,6 anos, com idade mínima de 17 e máxima de 53 anos. Segundo o estado civil, observa-se que $70 \%$ são solteiros enquanto $25 \%$ são casados e $3 \%$ divorciados. A maior parte da amostra (80\%) relaciona-se ao gênerofeminino e quase metade dela (47\%), trabalha além de estudar. Em relação ao gênero masculino, 76,9\% destes trabalham, além de estudar. 
Dos estudantes que trabalham, 45\% possuem jornada de trabalho acima de 40 horas/semana e 27,4\% com 30 a 40 horas/semana. Quanto à profissão, 40\% dos estudantes são auxiliares de enfermagem e 11\% técnicos de enfermagem.

De acordo com o local de residência, 79\% residem em Maringá, enquanto 20\% são de outras cidades. Dos estudantes, 133 (69,3\%) vivem com a família e $54(27,6 \%)$ longe dos pais.

Os dados foram agrupados por alguns componentes que permeiam o estilo de vida com suas respectivas variáveis. Para facilitar o entendimento dos componentes do estilo de vida optou-se por apresentá-los em forma de tabelas.

\section{Componente: Hábitos Alimentares (n=192 e p=100\%)}

Tabela 1 - Distribuição percentual e absoluta por hábitos alimentares de estudantes de enfermagem de uma instituição do sul do Brasil. Maringá/PR, 2006.

\begin{tabular}{|c|c|c|}
\hline Hábitos alimentares & $\mathbf{n}$ & $\%$ \\
\hline \multicolumn{3}{|l|}{ Tipos de alimentos ingeridos } \\
\hline Comer verduras e frutas & 19 & 9,9 \\
\hline Comer verduras e massa & 7 & 3,7 \\
\hline Comer apenas massa & 6 & 3,1 \\
\hline Comer frutas e massa & 5 & 2,6 \\
\hline Comer apenas grãos* & 9 & 4,7 \\
\hline Comer verduras e grãos & 19 & 9,9 \\
\hline Comer massa e grãos & 6 & 3,1 \\
\hline Ingesta equilibrada** & 111 & 57,8 \\
\hline 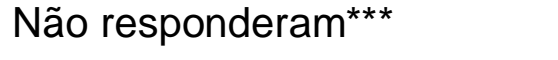 & 10 & 5,2 \\
\hline \multicolumn{3}{|c|}{ Frequência de almoço/ semana } \\
\hline Nenhuma & 15 & 7,8 \\
\hline Uma a três refeições & 23 & 12 \\
\hline Quatro a sete refeições & 141 & 73,4 \\
\hline Não responderam & 13 & 6,8 \\
\hline \multicolumn{3}{|c|}{ Frequência de lanches/ semana } \\
\hline Nenhuma & 13 & 6,8 \\
\hline Raramente & 8 & 4,2 \\
\hline Uma a três vezes & 88 & 45,8 \\
\hline Quatro a sete vezes & 47 & 24,5 \\
\hline Maior que sete & 5 & 2,6 \\
\hline Não responderam & 31 & 16,1 \\
\hline
\end{tabular}

*Grãos: arroz, feijão e/ou milho.

**Ingesta equilibrada: a combinação de todos os elementos acima descritos (verduras, frutas, massas e grãos).

${ }^{\star * *}$ Não responderam ao item ou a resposta estava incompleta e, portanto, considerada insuficiente. 
Quanto aos hábitos alimentares, 111 (57,8\%) apresentam um padrão adequado, contendo grande variedade de componentes essenciais para um bom equilíbrio nutricional (verduras, frutas, grãos e massa). Entretanto, é seguido por dois grupos - aproximadamente 10\% - de alimentos com pouca variedade nutricional (apenas "verduras e frutas" ou "verduras e grãos"), ingeridos pelos estudantes.

O fato de ingerirem, durante as refeições, pouca variedade e, especificamente, alimentos pouco calóricos, possivelmente tem relação com a prática de seguirem alguma dieta. Pode-se inferir que há, nesses estudantes, uma supervalorização do corpo influenciada pela mídia e a busca por este padrão de beleza (MONTEIRO et al, 2009). A necessidade de seguirem esse tipo de padrão alimentar pode estar relacionada ao fato de serem predominantemente estudantes do sexo feminino.

Em relação à frequência da alimentação observa-se que, ao considerar os estudantes que não tem prática alguma em almoçar ou aqueles que almoçam apenas uma a três vezes/ semana tem-se uma proporção de 38 (19,8\%). Dos estudantes que têm o hábito de lanchar, percebe-se que $88(45,8 \%)$ o fazem de uma a três vezes/ semana e $47(24,5 \%)$ de quatro a sete vezes. Ao serem somados, chega-se a um resultado preocupante sobre o hábito alimentar desses estudantes.

Acredita-se que, devido ao fato de muitos estudantes serem originalmente de outras cidades e por não residirem com os pais, eles tiveram menor frequência no consumo de todos os componentes alimentícios importantes para uma alimentação equilibrada. Isto pode ser justificado pelas limitações ao acesso, compra e preparo dos alimentos ou até mesmo por não poderem se alimentar constantemente em restaurantes devido à escassez de recursos financeiros. Prover a própria alimentação é uma experiência nova que demanda oportunidade, dedicação e disputa de tempo com as atividades estudantis e, muitas vezes, o estudante prioriza o sucesso acadêmico em detrimento da própria saúde (ALVES e BOOG, 2007).

\section{Componente: Atividade Física (n=192 e p=100\%)}

Tabela 2 - Distribuição percentual e absoluta das atividades físicas de estudantes de enfermagem de uma instituição do sul do Brasil. Maringá/PR, 2006.

\begin{tabular}{lll}
\multicolumn{1}{c}{ Atividade Física } & $\mathrm{n}$ & $\%$ \\
\hline Prática de atividades física & & \\
Sim & 77 & 40,1
\end{tabular}


Não

Não responderam*
$104 \quad 54,2$

$11 \quad 5,7$

\section{Tipos de atividade}

Esportes**

Atividades físicas não-competitivas ${ }^{\star \star \star}$
$21 \quad 27,3$

$38 \quad 49,4$

$18 \quad 23,3$

* Não responderam ao item ou a resposta estava incompleta e, portanto, considerada insuficiente.

**São atividades competitivas: Futebol, Futsal, Volei, Natação, Tênis, Luta.

*** Dança, Ginástica, Musculação, Hidroginástica, Caminhada.

${ }^{\star * \star \star}$ Estudantes que praticam atividades competitivas e não-competitivas.

Ao analisar as respostas dos estudantes com relação aos seus hábitos de atividade física pode-se constatar que a maioria deles, 104 (54,2\%) não pratica atividade física. Dentre os estudantes que praticam, a metade deles, aproximadamente $38(49,4 \%)$ realiza atividades não-competitivas.

Estudos similares com estudantes universitários apresentam inquietações e preocupações quanto ao lugar que a atividade física tem no estilo de vida desse grupo populacional. Morales e Souto (2006) verificaram que em uma amostra dos estudantes da Universidade da Região de Joinville-SC, cerca de 38\% dos estudantes não realizavam atividades físicas, ou seja, isto torna mais preocupante ainda o resultado $(54,2 \%)$ encontrado neste estudo.

A ausência de atividade física entre estudantes da área da saúde, mesmo possuindo conhecimento sobre sua importância e necessidade, parece ser uma constante. Em uma pesquisa realizada em uma Universidade de Minas Gerais, a fim de se conhecer o entendimento sobre o estilo de vida de estudantes de enfermagem e possíveis mudanças durante o período letivo, observou-se que houve alterações positivas no componente alimentação, mas não na atividade física que decaiu durante o curso (SOARES e CAMPOS, 2008).

Diante disso, torna-se essencial atentar a este grupo, visto que as evidências que associam hábitos satisfatórios de saúde a prática de atividade física estão bem documentados pela comunidade científica e parece haver um consenso de que o componente "atividade física" se destaca como um dos aspectos mais importantes para um estilo de vida saudável (NAHAS, 2003; SANTOS e ALVES, 2009).

\section{Componente: Padrão de Sono/Repouso (n=192 e p=100\%)}

Tabela 3 - Distribuição percentual e absoluta do padrão de sono/repouso de estudantes de enfermagem de uma instituição do sul do Brasil. Maringá/PR, 2006. 


\begin{tabular}{lcc}
\hline Padrão sono/repouso & $\mathbf{n}$ & \% \\
\hline Uma a três horas/ dia & 4 & 2,1 \\
Quatro a sete horas/ dia & 107 & 55,7 \\
Oito a dez horas/ dia & 70 & 36,5 \\
Dias alternados* & 5 & 2,6 \\
Não responderam $^{\star *}$ & 6 & 3,1 \\
\hline
\end{tabular}

*Sistema de plantões de estudantes-trabalhadores na área hospitalar.

** Não responderam ao item ou a resposta estava incompleta e, portanto, considerada insuficiente.

Quanto ao padrão sono/repouso nota-se que, a maior proporção 111 (57,8\%) dos estudantes dorme menos de sete horas/ dia. Isto se deve ao fato de ser grande a proporção de estudantes-trabalhadores no curso de enfermagem, além de serem predominantes os trabalhadores na área da enfermagem (técnicos e auxiliares de enfermagem), enfrentando muitas vezes duplas ou triplas jornadas de trabalho.

Estudos similares demonstram que os estudantes de enfermagem dormem menos de 8 horas de sono consideradas ideais por noite, apresentando um sono insuficiente e de baixa qualidade (PIZÃO; MARTINO, 2005; IGLESIAS, 2002). Os autores acrescentam que, os estudantes que trabalham no período noturno (plantões) dormem cerca de 05h13min/ noite durante a jornada, trabalhando conforme esquema de plantões noturnos de 12 horas, não sendo sempre possível dormir durante a jornada de trabalho ou depois da mesma e fazendo com que os estudantes-trabalhadores durmam menos que os que não trabalhavam.

Assim sendo, observando o resultado apresentado neste estudo, é possível perceber que, durante a semana, estes estudantes dormem tarde e acordam cedo, motivo este considerado a causa provável do déficit de sono, que vai se acumulando no decorrer da semana, causando um sono noturno de baixa qualidade e uma sensação de persistência do cansaço ao acordar. Pode-se ainda inferir que toda essa problemática interfere no padrão de sono dos estudantes, podendo afetar o estilo e a qualidade de vida e o seu processo de aprendizado.

\section{Componente: Consumo de tabaco $(n=192$ e $p=100 \%)$}

Tabela 4 - Distribuição percentual e absoluta do consumo de tabaco por estudantes de enfermagem de uma instituição do sul do Brasil. Maringá/PR, 2006.

\begin{tabular}{|c|c|c|}
\hline Consumo de tabaco & $\mathbf{N}$ & $\%$ \\
\hline Uso de tabaco & & \\
\hline Sim & 19 & 9,9 \\
\hline Não & 161 & 83,8 \\
\hline
\end{tabular}


Não responderam*

\section{Frequência do uso}

Dois a cinco cigarros/ dia

Seis a dez cigarros/ dia

Um masso/ dia

Dois a três massos/ semana
12

6,3

* Não responderam ao item ou a resposta estava incompleta e, portanto, considerada insuficiente.

Em relação aos hábitos tabágicos, a minoria, ou seja, 19 (9,9\%) eram de estudantes fumantes. Pode-se considerar positivo esse resultado de mais de $80 \%$ de não-fumantes, entre todos os respondentes. Outro pormenor importante a investigar era a quantidade de cigarros fumados pelos estudantes. A maioria dos estudantes fumantes, ou seja, 52,6\% fumavam de 2 a 5 cigarros/ dia, e 26,3\% fumavam de 1 a 3 massos de cigarros/ semana.

A constatação de que futuros profissionais da área de saúde usem tabaco tem sido motivo de preocupação por parte dos pesquisadores. Em um estudo realizado com estudantes de enfermagem de uma Universidade federal do interior de Minas Gerais foi verificado que, dentre os estudantes entrevistados 85\% consideram incoerente o enfermeiro fumar. Esse aspecto é visto como um problema pelos estudantes uma vez que o enfermeiro trabalha com questões como a prevenção, a promoção e o cuidado com a saúde (SANTOS, RODRIGUES e REINALDO, 2007).

Segundo um estudo realizado entre os estudantes de enfermagem da Universidade Estadual do Rio de Janeiro, os principais fatores que levam os alunos a adquirirem e a manterem um comportamento de risco são a curiosidade e o desejo de fumar, em primeiro lugar, seguida da influência do grupo extra familiar e da ajuda para aliviar as tensões do trabalho e dos estudos (MATSUMOTO, et al., 2005).

Entretanto, o estudante fumante que leva este hábito inadequado para seu futuro profissional, poderá deparar-se com situações conflitantes por ocasião da assistência prestada em ambiente de trabalho, independente da área em que atuará como enfermeiro; principalmente, na relação de confiança que deve existir entre o binômio profissional-paciente (TAUIL, COELHO e MONTEIRO, 2006).

Os profissionais da área de saúde são modelos a serem seguidos pela população em geral, e são eles que poderão determinar a efetividade ou não das políticas de saúde que visam a prevenção primária de doenças bem como o êxito de 
estratégias direcionadas ao controle do tabagismo em instituições de saúde (TAUIL, COELHO e MONTEIRO, 2006).

Componente: Consumo de álcool (n=192 e p=100\%)

Tabela 5 - Distribuição percentual e absoluta do consumo de álcool por estudantes de enfermagem de uma instituição do sul do Brasil. Maringá/PR, 2006.

\begin{tabular}{lcc}
\hline \multicolumn{1}{c}{ Consumo de álcool } & $\mathrm{N}$ & $\%$ \\
\hline Uso de álcool & & \\
Sim & 52 & 27,1 \\
Não & 127 & 66,1 \\
Não responderam* & 13 & 6,8
\end{tabular}

\section{Frequência do uso}

Apenas finais de semana $\quad 12 \quad 23,1$

Uma a três vezes/ semana $\quad 17 \quad 32,7$

Quatro a cinco/ semana $\quad 5 \quad 9,6$

Ocasionalmente**$\quad 18 \quad 34,6$

* Não responderam ao item ou a resposta estava incompleta e, portanto, considerada insuficiente.

** Relativo a beber "socialmente".

Dentre os $52(27,1 \%)$ estudantes etilistas, 22 (42,3\%) o fazem de 1 a 5 vezes/ semana, considerados bebedores moderados a graves, o que torna preocupante este perfil do estilo de vida, uma vez que há responsabilidades em seu cotidiano acadêmico como: acordar cedo para as aulas, estágios curriculares e até mesmo extra-curriculares, além de trabalhos acadêmicos a serem realizados.

Em pesquisa realizada entre estudantes de enfermagem da Universidade Estadual do Rio de Janeiro evidenciou que, o consumo frequente do álcool resulta no enfraquecimento nas relações interpessoais, déficit do rendimento acadêmico, tal como: dificuldade de concentração, atraso às aulas (geralmente no dia seguinte às festas/baladas), distúrbio de humor, alterando assim o estilo e qualidade de vida além do desenvolvimento acadêmico (MARÇAL, ASSIS e LOPES, 2005).

Os motivos referidos pelos estudantes em relação à ingestão de álcool geralmente estão associados ao lazer, ou seja, momentos de diversa/descontração, em primeiro lugar, seguido da influência do meio social, além de fatores emocionais impulsionados por problemas familiares (MARÇAL, ASSIS e LOPES, 2005).

Assim sendo, pode-se inferir que o meio universitário altera o estilo de vida do estudante de enfermagem estimulando-o abusar do álcool devido fatores como festas constantemente promovidas para obter recursos para a formatura, bem como 
a necessidade de socialização para alívio das tensões adquiridas no decorrer do curso.

\section{CONCLUSÃO}

A partir dos resultados obtidos, acredita-se que o objetivo inicialmente proposto foi alcançado. Assim sendo, pode-se concluir que os estudantes analisados no presente estudo não apresentam um estilo de vida saudável, o que os coloca em situação de risco para o desenvolvimento de vários agravos a saúde.

Em relação aos hábitos alimentares, mostra-se preocupante a inadequada ingesta alimentar, levando-os a um aumento dos riscos de carência nutricionais, uma vez que se trata de futuros profissionais enfermeiros que serão formadores de opinião sobre práticas alimentares e hábitos de vida saudáveis para a população.

Segundo os hábitos de atividade física, é preocupante a proporção de estudantes que não realizam nenhuma atividade, e dos que praticam, exercem atividades físicas não-competitivas o que inclui exercícios neuromusculares, talvez em decorrência da faixa etária, pode estar relacionado a motivadores estéticos.

A cerca do padrão de sono/repouso, nota-se que os estudantes de enfermagem possuem qualidade do sono ruim, podendo estar relacionada a irregularidade do sono, menor duração, comprometimento na eficiência e preocupações com notas e práticas de ensino.

De acordo com os comportamentos de risco como o tabaco e o álcool, acredita-se que a promoção de medidas que contribuam para a redução ou mesmo a eliminação da prática e/ou hábitos inadequados no ambiente acadêmico, contribuirá para a melhor o estilo e qualidade de vida desses futuros profissionais bem como a promoção de ambientes saudáveis.

Sugere-se a elaboração de um programa de promoção de saúde na instituição, a ser implementado de forma efetiva em conjunto com o departamento de Educação Física, a fim de ofertar de forma ampla, diferentes atividades físicas extracurriculares, com a finalidade de atender aos interesses e necessidades não só dos estudantes de enfermagem como também de outros cursos. Alem disso, a elaboração de ferramentas virtuais de avaliação e aconselhamento seria de grande auxílio para os estudantes na tomada de decisões a respeito de um estilo de vida saudável. 


\section{REFERÊNCIAS}

ALVES, H. J; BOOG, M. C. F. Comportamento alimentar em moradia estudantil: um espaço para promoção da saúde. Revista de Saúde Pública, São Paulo, v. 41, n. 2, p. 197-204, 2007.

BRASIL. Ministério da Saúde (MS). Secretaria de Assistência à Saúde. Departamento de Assistência e Promoção da Saúde. Coordenação de Saúde Mental. Serviço de Atenção ao Alcoolismo e Drogadição. Normas e Procedimentos na abordagem do alcoolismo. 2. Ed. Brasília: MS; 1994.

COELHO, C. W; SANTOS, J. F. S. Perfil do estilo de vida relacionado à saúde dos calouros de um centro de ciências tecnológicas. Revista Digital, Buenos Aires, v. 11 , n. 97, Junho de 2006.

FRANCA, C; COLARES, V. Estudo comparativo de condutas de saúde entre universitários no início e no final do curso. Rev. Saúde Pública, v. 42, n. 3, p. 420427, 2008.

IGLESIAS, R. B. Qualidade de vida de alunos-trabalhadores que cursam a graduação em enfermagem. 2002. 97f. Dissertação (Mestrado em Enfermagem) Escola de Enfermagem, Universidade de São Paulo, São Paulo.

JOIA, L. C. Perfil do estilo de vida individual entre estudantes universitários. Revista Movimenta, v. 3, n. 1, 2010.

MARÇAL, C. L. A; ASSIS, F; LOPES, G. T. O uso de bebidas alcoólicas pelos estudantes de enfermagem da Universidade do Estado do Rio de Janeiro (FENF/UERJ). Revista SMAD, v. 1, n. 2, p. 1-16, 2005.

MATSUMOTO, K. S. et al. O uso do tabaco entre os universitários de enfermagem da universidade do estado do Rio de Janeiro (UERJ). Revista SMAD, v. 1, n. 2, p. 114, 2005.

MONTEIRO, M. R. P. et al. Hábito e consumo alimentar de estudantes do sexo feminino dos cursos de nutrição e de enfermagem de uma universidade pública brasileira. Rev. APS, v. 12, n. 3, p. 271-277, jul./set. 2009.

MORAlES, P. J. C; SOUTO, V. I. V. A Prática da Atividade Física no Meio Acadêmico da Univille, de Joinville, SC. In: $5^{\circ}$ Fórum Internacional de Qualidade de Vida e Saúde, 2006, Curitiba. Revista Científica do Fórum Internacional de Qualidade de Vida e Saúde. Curitiba/PR: Editora Korpus, v. 01. p. 186-188, 2006.

NAHAS, M. V. Atividade Física, Saúde e Qualidade de Vida: Conceitos e Sugestões para um Estilo de Vida Ativo. 3. ed. Londrina - PR: Midiograf. 2003. 
OGUISSO, T; SEKI, L. K. A prevalência do tabagismo entre estudantes de graduação da Escola de Enfermagem da Universidade de São Paulo. Rev Esc En USP, v. 35, n. 1, p. 19-27, mar. 2001.

PIZÃO, M. F.; MARTINO, M. M. F. Estudo do sono em estudantes universitários de enfermagem. 2005. In: XIII CONGRESSO INTERNO DE INICIAÇÃO CIENTÍFICA DA UNINCAMP - GINÁSIO MULTIDISCIPLINAR DA UNINCAMP, set., 2005. Campinas. Anais... Campinas: UNINCAMP, 2005.

SANTOS, J. F. S. Estilo de vida e percepção de qualidade de vida de acadêmicos de ciências tecnológicas: um estudo de caso. In: Anais do II congresso científico latinoamericano da FIEP. Foz do Iguaçu. Anais... Foz de Iguaçu: FIEP, 2004.

SANTOS, J. F. S; ALVES, V. S. Perfil do estilo de vida relacionado à saúde dos acadêmicos da Unicentro, Campos Irati, PR. Revista Digital, Buenos Aires, v. 13, n. 129, Fevereiro de 2009.

SANTOS, K. P, RODRIGUES, A; REINALDO, M. A. S. Relação entre a formação acadêmica dos estudantes de enfermagem e sua percepção quanto ao tabagismo. Rev. Eletr. Enf., v. 9, n. 2, p. 432-42, 2007.

SOARES, R. D. O. P; CAMPOS, L. F. Estilo de vida dos estudantes de enfermagem de uma universidade do interior de minas gerais. Cogitare Enferm., v. 13, n. 2, p. 227-34, Abr/Jun 2008.

TAUIL, M. C; COELHO, R. A. C; MONTEIRO, P. S. Prevalência do uso de fumo entre alunos do curso de graduação em enfermagem da Universidade de Brasília. Comun Ciênc Saúde. v. 17, n. 2, p. 121-127, 2006.

UCHOA, E; ROSEMBERG, B; PORTO, M. F. S. Entre a fragmentação e a integração: saúde e qualidade de vida de grupos populacionais específicos. Informe epidemiológico do SUS, v. 11, n. 3, p. 115-28, 2002.

VIEIRA, V. C. R. et al. Perfil socioeconômico, nutricional e de saúde de adolescentes recém-ingressos em uma universidade pública brasileira. Rev. Nutr., Campinas, v. 15, n. 3, p. 273-82, 2002. 